\title{
Laser frequency offset locking using electromagnetically induced transparency
}

\author{
S. C. Bell and D. M. Heywood \\ Optical Physics, University of Melbourne, Victoria 3010, Australia \\ J. D. White \\ Juniata College, Huntingdon, Pennsylvania 16652 \\ J. D. Close \\ Australian National University, Canberra ACT 0200, Australia \\ R. E. Scholten ${ }^{\text {a) }}$ \\ School of Physics, University of Melbourne, Victoria 3010, Australia
}

(Received 20 February 2007; accepted 2 April 2007; published online 27 April 2007)

\begin{abstract}
The authors have used an electromagnetically induced transparency resonance in rubidium as a dispersive reference to lock the relative frequency of two lasers to the atomic ground-state hyperfine splitting. The beat frequency between the two lasers directly generates a microwave signal at $3.036 \mathrm{GHz}\left({ }^{85} \mathrm{Rb}\right)$ or $6.835 \mathrm{GHz}\left({ }^{87} \mathrm{Rb}\right)$. High bandwidth $(600 \mathrm{kHz})$ feedback was achieved with only low-frequency $(10 \mathrm{MHz})$ electronics using the frequency modulation sideband method. The spectral width of the microwave beat frequency was reduced to less than $1 \mathrm{kHz}$. The technique offers a convenient and low-cost method suitable for many topical two-frequency experiments, including coherent population trapping, slow light, lasing without inversion, and Raman sideband cooling. () 2007 American Institute of Physics. [DOI: 10.1063/1.2734471]
\end{abstract}

Many interesting laser-atom interactions require two laser beams with a fixed frequency difference related to atomic energy level splittings. Examples include atomic coherence effects in the alkali atoms, such as slow light, lasing without inversion, electromagnetically induced transparency, and atomic clocks. ${ }^{1}$ Laser cooling and trapping of alkalis require a repump laser to prevent dark-state losses, and Raman sideband cooling specifically relies on two lasers with precise offset frequency. ${ }^{2}$

The two frequency components needed for experiments can be generated from a single laser beam, with a modulator (acousto-optical $^{3}$ or electro-optical ${ }^{4}$ ), or by direct modulation of the laser current for diode lasers.

Alternatively, two separate lasers can be used, individually referenced to atomic transitions ${ }^{6}$ or to Fabry-Pérot etalon fringes, but in many experiments only the frequency difference must be controlled to high precision, while the absolute frequency is less important. The difference can be stabilized by offset locking the frequency of one laser relative to the other using the interference beat between the two. Offset locking requires electronics operating at the offset frequency, ${ }^{7,8}$ typically in the microwave (gigahertz) for ground-state alkali atoms and diamond nitrogen-vacancy centers. ${ }^{9}$ Microwave detectors, precision synthesized oscillators, and associated high-frequency electronics provide broad tunability, but can be expensive and complex, and the frequency difference is not intrinsic to the atomic system of interest.

An electromagnetically induced transparency (EIT) resonance provides a direct relative frequency reference. Using an EIT resonance, only low-frequency detectors and servo electronics are needed to lock the frequency difference between two lasers to an atomic hyperfine splitting. EIT reso-

\footnotetext{
${ }^{a)}$ Electronic mail: scholten@unimelb.edu.au
}

nances can be much narrower than natural transition linewidths, with greater dispersion and the potential for narrower relative frequency uncertainty. In early demonstrations, ${ }^{10,11}$ absorption-based detection of the EIT signal limited the feedback bandwidth to a fraction of the natural spontaneous linewidth $\Gamma$ ( $6 \mathrm{MHz}$ for $\mathrm{Rb})$, and the relative laser spectral width achieved was similar to that of individually locked lasers, $\sim 100 \mathrm{kHz}$.

In our experiments, we use the frequency modulation (FM) sideband method, ${ }^{12}$ modulating the laser frequency at $10 \mathrm{MHz}$ to generate a feedback signal from the relative phase difference of the atomic vapor at the $\pm 10 \mathrm{MHz}$ sidebands above and below an EIT resonance. The feedback bandwidth limit is then determined by the modulation frequency ${ }^{13}$ rather than the resonance width, and in our case, is greater than the linewidth of each individual laser, allowing line narrowing to achieve a noise-limited relative frequency spectral width below $1 \mathrm{kHz}$.

Figure 1 shows the relevant energy levels of rubidium and the photodetector signal as the probe laser was scanned through the EIT resonance. A narrow EIT peak (width

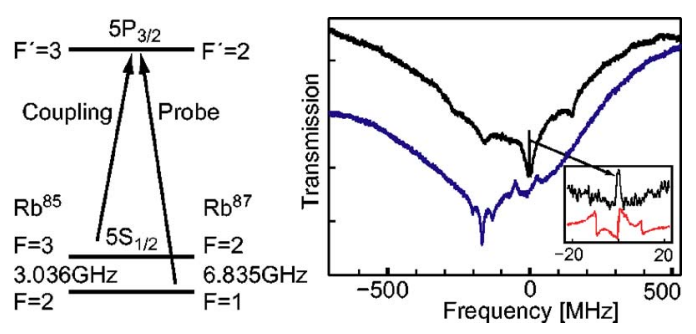

FIG. 1. (Color online) Rubidium energy levels, ${ }^{87} \mathrm{Rb}$ EIT spectrum (upper), and $F=1$ ground-state saturated absorption spectrum for probe laser (lower). The inset shows expanded EIT region with FM sideband feedback error signal. EIT peak height $30 \%$ of Doppler absorption; width of $0.85 \mathrm{MHz}$ limited by linewidth of unlocked (scanning) probe laser. 


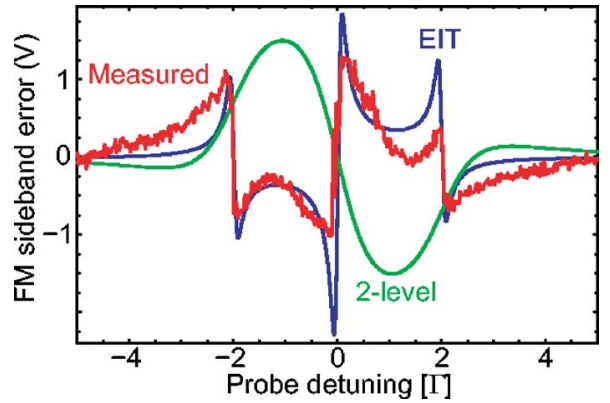

FIG. 2. (Color online) Measured FM sideband error signal for the EIT resonance compared to calculations for EIT and an equivalent two-level Doppler-free system. Opposite signs of error curves reflect sign reversal in refractive index for two- and three-level systems, calculated for strong coupling, weak probe, Rabi frequencies 2 and $0.01 \Gamma$, and modulation frequency $2 \Gamma$.

$<1 \mathrm{MHz}$ ) was observed when an atomic coherence was induced between the two atomic hyperfine ground states via a common excited state. The coupling laser was locked to a saturated absorption crossover peak, either $F=2 \rightarrow F^{\prime}$ $=(2,3)$ for ${ }^{87} \mathrm{Rb}$ or $F=3 \rightarrow F^{\prime}=(3,4)$ for ${ }^{85} \mathrm{Rb}$. Atoms with appropriate velocity in the direction of laser propagation were resonant on the $F=2 \rightarrow F^{\prime}=2$ transition. The probe was tuned to $F=1 \rightarrow F^{\prime}=2$ for atoms at the same velocity. The EIT resonance occurs where the frequency difference between the coupling and probe lasers precisely matches the ground-state hyperfine splitting, independent of fluctuations of the coupling laser frequency. Thus, as the coupling laser frequency changes, so too does the offset-locked probe, but their difference is maintained.

In Fig. 2, the EIT dispersion is compared with that for a conventional two-level Doppler-free saturated absorption signal. The calculated values are from an optical Bloch equation model $^{14}$ for the three-level two-laser $\Lambda$-configuration EIT system, solved for steady-state conditions using matrix inversion and averaged over appropriate Doppler detunings and weighting for a Maxwellian velocity distribution. The figure shows the calculated FM sideband error signal, a measured error signal, and the prediction for a two-level atom calculated by reducing the coupling laser power to a negligible value. The dispersion is clearly much greater at resonance for the EIT system, consistent with the narrow width of the EIT resonance relative to the natural linewidth.

The experimental setup is shown in Fig. 3. The two external cavity diode laser ${ }^{15}$ beams were coupled into a singlemode fiber and copropagated through a room-temperature magnetically shielded rubidium cell. The coupling laser

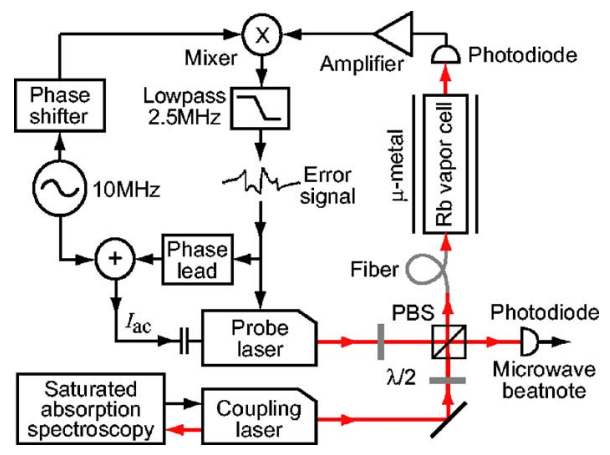

FIG. 3. (Color online) Experimental setup for frequency offset-locking us-

ing EIT. PBS polarizing beam splitter; $\lambda / 2$ half-wave retarder. horizontal; $5 \mathrm{~dB} /$ div vertical.
Downloaded 21 May 2008 to 130.56 .17 .236 . Redistribution subject to AIP license or copyright;

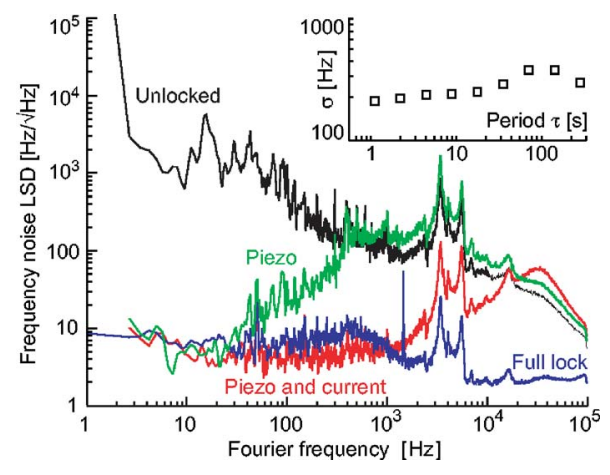

FIG. 4. (Color online) Frequency noise linear spectral density of feedback error signal; coupling laser locked, different probe laser feedback actuators enabled. Noise suppression extends to $600 \mathrm{kHz}$ (not shown). Inset: overlapping Allan standard deviation $\sigma(\tau)$ of the offset beat note frequency.

power was $2 \mathrm{~mW}$, with $1 / e^{2}$ radius of $2 \mathrm{~mm}$, and linewidth of $300 \mathrm{kHz}$. The diode injection current of the probe laser (power of $1 \mathrm{~mW}$ ) was modulated at $10 \mathrm{MHz}$. The combined probe and coupling lasers were incident on a Si $p-i-n$ photodiode. The detector current was amplified and mixed with the phase-shifted reference, and the higher harmonics removed with a $2.5 \mathrm{MHz}$ low-pass filter. The error signal (Fig. 2) was fed back to the probe laser to stabilize the coupling-probe frequency difference at the ground-state hyperfine splitting.

The probe laser frequency was controlled via two actuators and three feedback channels. A piezoelectric transducer provided feedback to the cavity length for Fourier frequencies up to $300 \mathrm{~Hz}$. A second channel controlled the diode injection current supply, ${ }^{16}$ with bandwidth of $20 \mathrm{kHz}$ limited by the diode thermal response which dominates at low Fourier frequencies. ${ }^{17}$ The error signal was also coupled directly to the diode via a capacitor and passive single-stage phaselead circuit, providing feedback at higher Fourier frequencies where the diode response is determined by the change in refractive index due to charge-carrier density modulation. ${ }^{17}$ Figure 4 shows the separate effects of these three feedback channels on the frequency noise spectrum of the locking signal.

The interference between the two laser beams on a fast $(7 \mathrm{GHz})$ photodiode produced a microwave beat note at the difference frequency. Figure 5 shows the rf spectrum of the beat note for ${ }^{87} \mathrm{Rb}$, with a $-3 \mathrm{~dB}$ width of $750 \mathrm{~Hz}$ for a $0.2 \mathrm{~s}$ sweep and $4 \mathrm{kHz}$ averaged over $22.5 \mathrm{~s}$. We measured the peak frequency once per second for $30 \mathrm{~min}$ to find an average of $6834649630 \mathrm{~Hz}$ with one standard deviation statis-

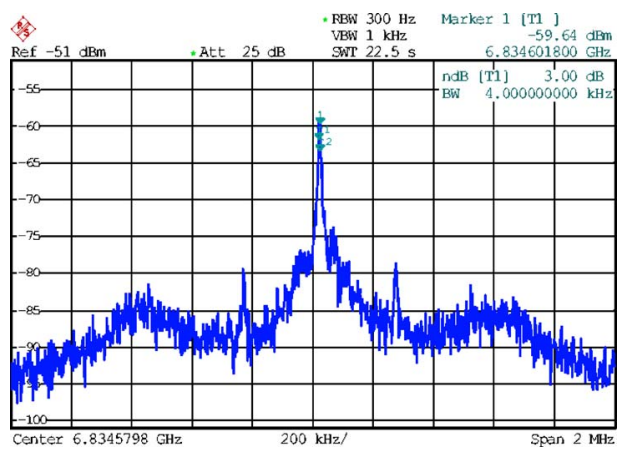

FIG. 5. (Color online) Spectrum of microwave beat note generated by interference for ${ }^{87} \mathrm{Rb} .-3 \mathrm{~dB}$ width of $4 \mathrm{kHz}(750 \mathrm{~Hz}$ in $0.2 \mathrm{~s}) .200 \mathrm{kHz} / \mathrm{div}$ horizontal; $5 \mathrm{~dB} /$ div vertical. 
tical uncertainty of $\pm 550 \mathrm{~Hz}$. The Allan variance ${ }^{18}$ of the data is shown in Fig. 4. The relative uncertainty of the beat frequency was below $5 \times 10^{-8}$, with a flat dependence on sampling time consistent with flicker frequency noise, as observed in some high precision oscillators including Cs clocks. ${ }^{19}$

The average frequency in this sample differed by $30 \mathrm{kHz}$ from the best available measurement, $6834682611 \mathrm{~Hz}{ }^{20}$ Systematic variations between different measurements were up to $\pm 50 \mathrm{kHz}$, thought to be caused by temperature-related drifts in the fiber coupling. The frequency was affected by both the absolute and relative intensities of the two beams, and their polarizations, due to ac Stark shift and optical pumping effects. ${ }^{14}$ We were able to adjust the beat frequency via a dc offset to the error signal, up to $\pm 250 \mathrm{kHz}$.

The broad shoulders on the beat note show the limited bandwidth of the diode response $(600 \mathrm{kHz})$. Small peaks at $\pm 250 \mathrm{kHz}$ are due to induced currents from the Zeeman modulation coil used for locking the coupling laser. ${ }^{15}$ Sidebands at the modulation frequency $(10 \mathrm{MHz})$ are more than $20 \mathrm{~dB}$ below the peak. The technique works equally well with ${ }^{85} \mathrm{Rb}$, for which we measured a microwave frequency of $3.03571 \mathrm{GHz}$.

In summary, we have combined a high bandwidth FM sideband frequency discriminator with a high-dispersion electromagnetically induced transparency resonance to lock two laser frequencies to an offset of the ground-state hyperfine splitting of rubidium. Our solutions to the three-level optical Bloch equations for a $\Lambda$-EIT system, convolved with the Maxwellian velocity distribution of a thermal vapor, support the relative benefits of the highly dispersive resonance compared to that of a two-level reference. We obtained a microwave beat note at $6.834650 \mathrm{GHz}$ for ${ }^{87} \mathrm{Rb}$ with linewidth and statistical frequency uncertainty below $1 \mathrm{kHz}$, using only low-frequency feedback electronics. For experiments which require frequency variability near the resonance, a low-frequency (megahertz) optical modulator could be used on one of the laser beams, without compromising the high bandwidth and stability of the method. We expect that the approach could be applied to other alkali atoms and other two-laser resonances, and that substantial improvements to the beat note stability and linewidth can be achieved, in particular, by reducing intensity drifts in the laser beams.

This research was supported under the Discovery funding scheme of the Australian Research Council (Project No. DP0557505).

${ }^{1}$ M. O. Scully and M. S. Zubairy, Quantum Optics (Cambridge University Press, Cambridge, 1997).

${ }^{2}$ H. J. Metcalf and P. van der Straten, Laser Cooling and Trapping (Springer-Verlag, New York, 1999).

${ }^{3}$ F. B. J. Buchkremer, R. Dumke, Ch. Buggle, G. Birkl, and W. Ertmer, Rev. Sci. Instrum. 71, 3306 (2000).

${ }^{4}$ M. Kasevich, D. S. Weiss, E. Riis, K. Moler, S. Kasapi, and S. Chu, Phys. Rev. Lett. 66, 2297 (1991).

${ }^{5}$ P. Feng and T. Walker, Am. J. Phys. 63, 905 (1995).

${ }^{6}$ K. Nakayama, M. Hyodo, R. Ohmukai, and M. Watanabe, Opt. Commun. 259, 242 (2006).

${ }^{7}$ R. L. Barger and J. L. Hall, Phys. Rev. Lett. 22, 4 (1969).

${ }^{8}$ U. Schünemann, H. Engler, R. Grimm, M. Weidemüller, and $M$. Zielonkowski, Rev. Sci. Instrum. 70, 242 (1999).

${ }^{9}$ C. Santori, P. Tamarat, P. Neumann, J. Wrachtrup, D. Fattal, R. G. Beausoleil, J. Rabeau, P. Olivero, A. D. Greentree, S. Prawer, F. Jelezko, and P. Hemmer, Phys. Rev. Lett. 97, 247401 (2006).

${ }^{10}$ A. M. Akulshin, A. A. Celikov, and V. L. Velichansky, Opt. Commun. 84, 139 (1991).

${ }^{11}$ H. S. Moon, L. Lee, K. Kim, and J. B. Kim, Appl. Phys. Lett. 84, 3001 (2004).

${ }^{12}$ G. C. Bjorklund, Opt. Lett. 5, 15 (1980).

${ }^{13}$ R. W. P. Drever, J. L. Hall, F. V. Kowalski, J. Hough, G. M. Ford, A. J. Munley, and H. Ward, Appl. Phys. B: Photophys. Laser Chem. 31, 97 (1983).

${ }^{14}$ L. P. Maguire, R. M. W. van Bijnen, E. Mese, and R. E. Scholten, J. Phys. B 39, 2709 (2006).

${ }^{15}$ C. J. Hawthorn, K. P. Weber, and R. E. Scholten, Rev. Sci. Instrum. 72, 4477 (2001).

${ }^{16}$ MOGlabs laser diode controller, www.moglabs.com

${ }^{17}$ S. Kobayashi, Y. Yamamoto, M. Ito, and T. Kimura, IEEE J. Quantum Electron. 18, 582 (1982).

${ }^{18}$ D. W. Allan, Proc. IEEE 54, 221 (1966); J. Rutman and F. L. Walls, ibid. 79, 952 (1991).

${ }^{19}$ F. Riehle, Frequency Standards: Basics and Applications (Wiley-VCH, Weinheim, 2004).

${ }^{20} 6834682610.90429(9) \mathrm{Hz}$; S. Bize, Y. Sortais, M. S. Santos, C. Mandache, A. Clairon, and C. Salomon, Europhys. Lett. 45, 558 (1999). 